\title{
Numerical Analysis of Loss Power Properties in the Near-Field Electromagnetic Wave Through A Microstrip Line for Multilayer Magnetic Films with Different Levels of Electrical Conductivity
}

\author{
Jung Hwan Lee and Sang Woo Kim* \\ Center for Energy Materials Research, Korea Institute of Science and Technology, Seoul 136-791, Korea
}

(Received 26 May 2008, Received in final form 18 September 2008, Accepted 24 September 2008)

\begin{abstract}
There are few reports of high frequency loss behavior in the near-field for magnetic films with semiconducting properties, even though semiconducting magnetic materials, such as soft magnetic amorphous alloys and nanocrystalline thin films, have been demonstrated. The electromagnetic loss behavior of multilayer magnetic films with semiconducting properties on the microstrip line in quasi-microwave frequency band was analyzed numerically using a commercial finite-element based electromagnetic solver. The large increase in the absorption performance and broadband characteristics of the semiconducting/insulating layer magnetic films examined in this study were attributed to an increase in the loss factor of resistive loss. The electromagnetic reflection increased significantly with increasing conductivity, and the loss power deteriorated significantly. The numerical results of the magnetic field distribution showed that a strong radiated signal on the microstrip line was emitted with increasing conductivity and decreasing film thickness due to re-reflection of the radiated wave from the surface of the magnetic film, even though the emitted levels varied with film thickness.
\end{abstract}

Key words : conductivity, multilayer film, electromagnetic wave absorber, microstrip line

\section{Introduction}

There has been considerable improvement in the development of information and communication technology with electronic devices and systems, such as notebooks, computers and mobile phones, becoming smaller, multi-functioned and highly integrated. According to technological trends, there are increasing problems with electromagnetic interference and electromagnetic compatibility (EMI/ EMC) in these devices or systems. One way of suppressing unwanted electromagnetic waves is to use a microwave absorber. In order to meet the requirements, the microwave absorber should be very thin but exhibit high performance at the higher resonant frequency. The microwave absorber absorbs unwanted electromagnetic waves from outer space and removes the reflected wave by dissipating the signal energy impinging on its surface in the form of heat. When the incident wave travels through a material, the higher absorption of the electromagnetic wave is due to the higher loss properties of electro-

*Corresponding author: Tel: +82-2-958-5526

Fax: +82-2-958-5529, e-mail: swkim@kist.re.kr magnetic materials. Several materials with a loss factor, such as conductive loss, dielectric loss, or magnetic loss, have been developed [1-3]. Among these materials, ferrite or soft magnetic metal alloy materials with magnetic loss have been examined for applications as microwave absorbers. In addition to these reports, studies of the high frequency loss behavior in the near-field for multilayer magnetic films with semiconducting properties are still in progress. In this study, the loss characteristics and noise distribution on a microstrip line (MSL) for semiconducting/insulating layer magnetic films with different thicknesses and conductivities were examined by numerical analysis using a commercial finite-element based electromagnetic solver.

\section{Experimental Procedure}

Numerical analysis was carried out using a microstrip line method in quasi-microwave band to examine the absorption properties of double layer magnetic films with semiconducting properties [4]. A high frequency structure simulator (HFSS) CAD simulation program based on a finite element method was used to simulate the electro- 


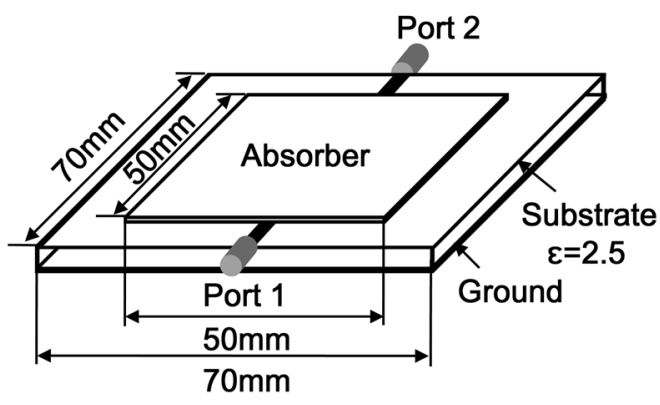

(a)

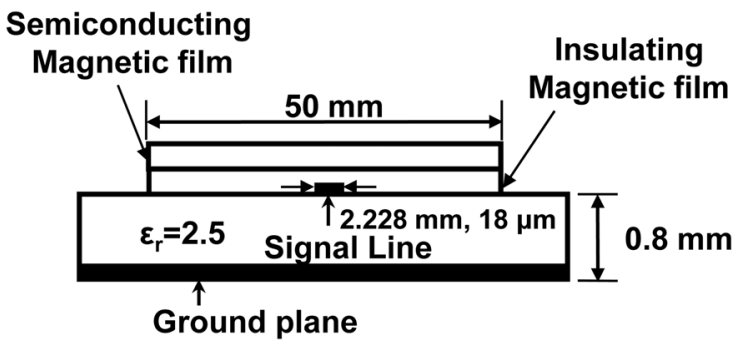

(b)

Fig. 1. (a) Simulation model of the microstrip line, and (b) its cross-sectional view.

magnetic loss properties and magnetic field distribution. Fig. 1 shows the device details for the simulation model. The microstrip line consisted of a copper conductor on a PTFE (poly(tetrafluoroethylene)) substrate, which is dielectric with a permittivity of 2.5 , and a ground at the bottom of the microstrip line. The signal line was 2.228 $\mathrm{mm}$ wide, $70 \mathrm{~mm}$ long and $0.012 \mathrm{~mm}$ thick, and the dimensions of the substrate in the designed microstrip line was $70 \times 70 \times 0.8 \mathrm{~mm}$. The reflection $\left(\mathrm{S}_{11}\right)$ and trans-

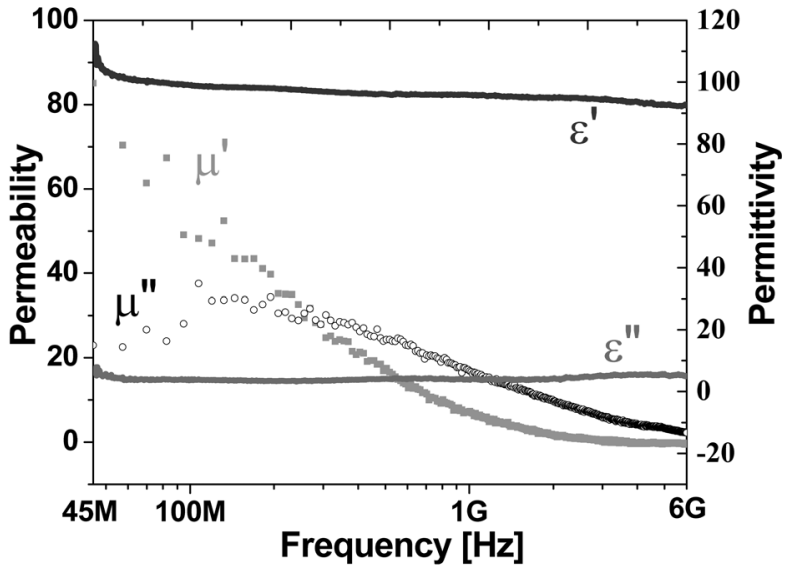

Fig. 2. Material properties of the semiconducting and insulating magnetic films.

mission $\left(\mathrm{S}_{21}\right)$ parameters of the microstrip line attached to the magnetic double layer films were calculated using the dielectric results obtained by the cavity perturbation methods at frequencies ranging from $100 \mathrm{MHz}$ to $6 \mathrm{GHz}$, where a film sample, $50 \mathrm{~mm}$ in length and width, was located at the center of the microstrip line with the characteristic impedance of $50 \Omega$.

Magnetic double layer films with a laminated structure of a semiconducting and insulating layer were used for the simulation. It was assumed that the conductivity of the insulating magnetic film was $8 \times 10^{-8} \mathrm{~S} / \mathrm{m}$ and the film was attached to the microstrip line with no gaps, as shown in Fig. 1(b). The semiconducting magnetic film with different conductivity was assumed to be layered on the insulating film and the thickness of each layer was half of the total thickness of the magnetic double layer

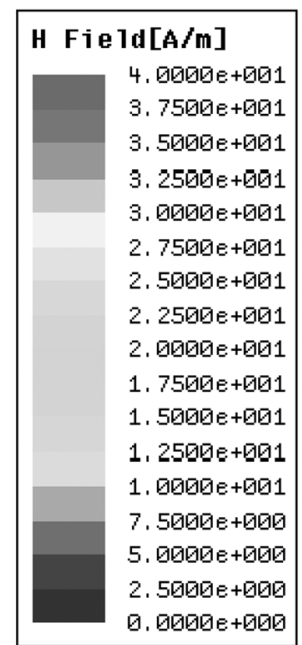

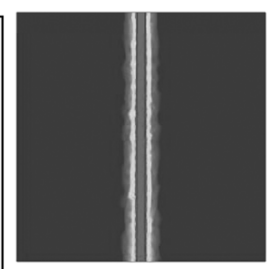

(a)

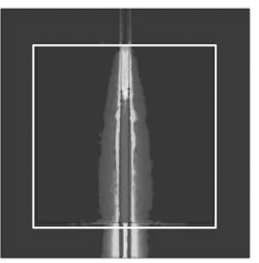

(d)

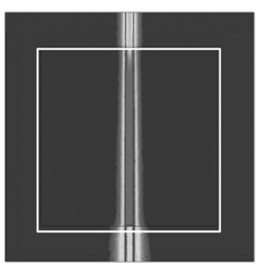

(b)

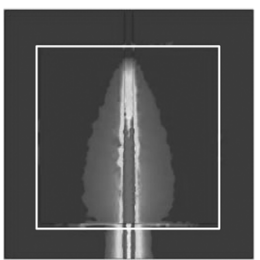

(e)

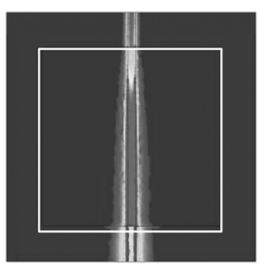

(c)

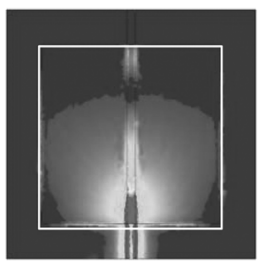

(f)

Fig. 3. The top view of the magnetic field distribution on the surface of the microstrip line attached to the semiconducting/insulating layered magnetic films with different conductivity at thickness of $50(25 / 25) \mu \mathrm{m}$. (a) Bare microstrip line and each conductivity (and reflection) is (b) $20 \mathrm{~S} / \mathrm{m}(5 \%)$, (c) $150 \mathrm{~S} / \mathrm{m}(20 \%)$, (d) $400 \mathrm{~S} / \mathrm{m} \mathrm{(38 \% ),} \mathrm{(e)} 10^{3} \mathrm{~S} / \mathrm{m}(50 \%)$, and (f) $10^{4} \mathrm{~S} / \mathrm{m}(70 \%)$, respectively. 
films. The conductivity and thicknesses of the semiconducting layer varied from $1 \mathrm{~S} / \mathrm{m}$ to $10^{5} \mathrm{~S} / \mathrm{m}$ and from 2 $\mu \mathrm{m}$ to $100 \mu \mathrm{m}$, respectively. Fig. 2 shows the frequency dependence of the material parameters of the semiconducting/insulating layer magnetic films.

\section{Results and Discussion}

Fig. 3 shows the top view of the magnetic field distribution on the surface of the microstrip line attached to the semiconducting/insulating layer magnetic film, 50 (25/25)

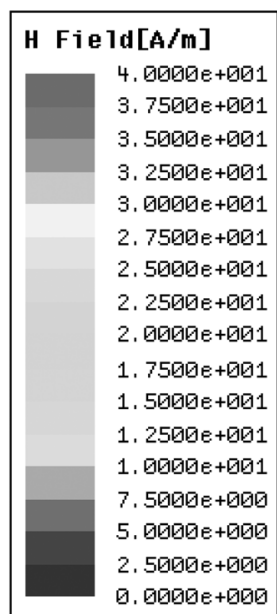

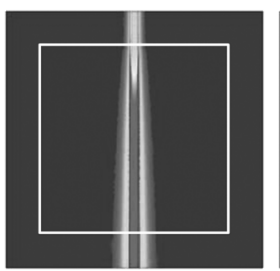

(a)

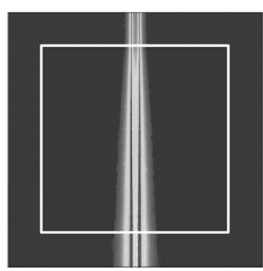

(e)

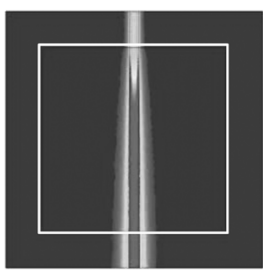

(b)

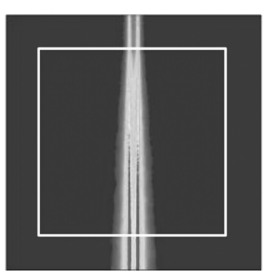

(f)

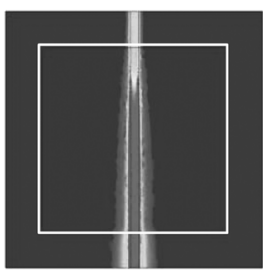

(c)

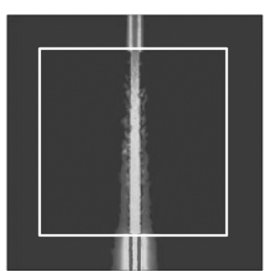

(g)

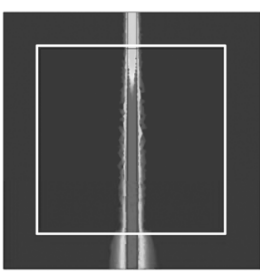

(d)

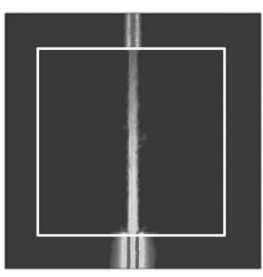

(h)

Fig. 4. The top view of the magnetic field distribution for the double layered films at various thicknesses; (a) 4 (2/2) $\mu \mathrm{m}$, (b) 10 (5/ 5) $\mu \mathrm{m}$, (c) $50(25 / 25) \mu \mathrm{m}$, and (d) $100(50 / 50) \mu \mathrm{m}$, respectively at $0.3 \mathrm{GHz}$, when all reflections of the double layered magnetic films were constant to approximately $20 \%$. (a)-(d) on the bottom surface of the magnetic film (i.e., on the surface of the microstrip line) and (e)-(h) on the top surface of the magnetic films.

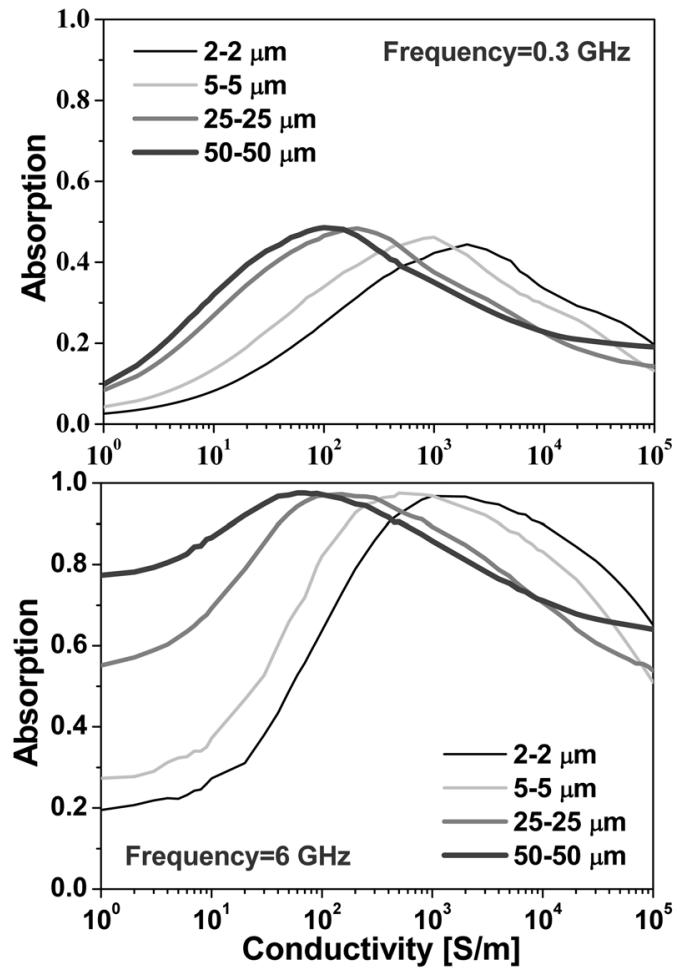

(a)
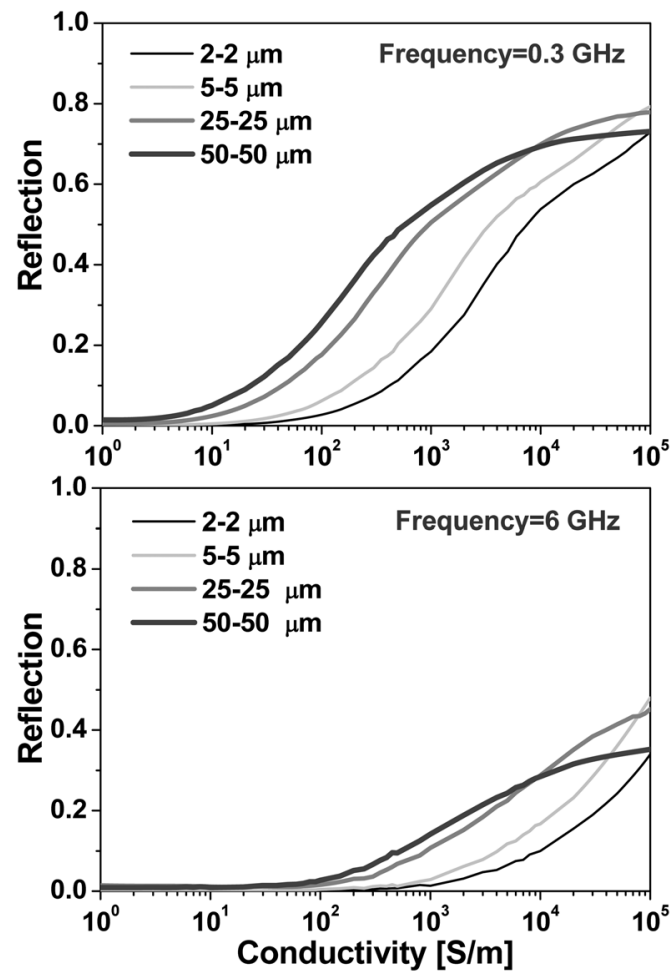

(b)

Fig. 5. The calculated (a) electromagnetic absorption and (b) reflection of the double layered magnetic films with different thicknesses at a reflection of $20 \%$ and frequencies of 0.3 and $6 \mathrm{GHz}$, respectively. 
$\mu \mathrm{m}$ thickness, with different conductivities (i.e., reflection). Strong radiated noise was generated when the reflection was $>20 \%(150 \mathrm{~S} / \mathrm{m})$, whereas radiated noise was not generated when the reflection was $<20 \%$. This was attributed to re-reflection from the surface of the magnetic films with conductivity $>150 \mathrm{~S} / \mathrm{m}$. These results suggest that the conductivity of the microwave absorber films need to be properly controlled because the magnetic absorber film with a reflection $>20 \%$ can generate radiation noise. Fig. 4 shows the top view of the magnetic field distribution on the bottom and top surfaces of the magnetic films at various thicknesses at $0.3 \mathrm{GHz}$, where all the reflections of the magnetic double layer films were constant to approximately $20 \%$. A top view of the bottom surface of the magnetic films showed that a radiated field was not generated for all films. The views on the top surface of the magnetic films indicated that the electromagnetic wave was effectively suppressed on the surface of the microstrip line and on the output port.

The numerical results in Fig. 5 show the reflection and absorption characteristics of the magnetic double layer films with different thicknesses at frequencies of 0.3 and $6 \mathrm{GHz}$, respectively. The maximum absorption at 0.3 and $6 \mathrm{GHz}$ increased up to $48 \%$ and $97 \%$, respectively. At $0.3 \mathrm{GHz}$, the absorption began to deteriorate with increasing conductivity at a reflection $>20 \%$ because the reflection increased up to $80 \%$ with increasing conductivity. At $6 \mathrm{GHz}$, there was a similar trend with increasing reflection but the decrease in absorption was observed at a reflection much lower than $20 \%$. This was attributed to the relatively high transmission and low reflection that was sufficient to be ignored in the region below $100 \mathrm{~S} / \mathrm{m}$ at high frequency, even though there was some dependence on film thickness. Fig. 6 shows the loss power characteristics of the magnetic double layer films with a reflection of $5 \%$ and $20 \%$. The power loss increased gradually with increasing frequency at both

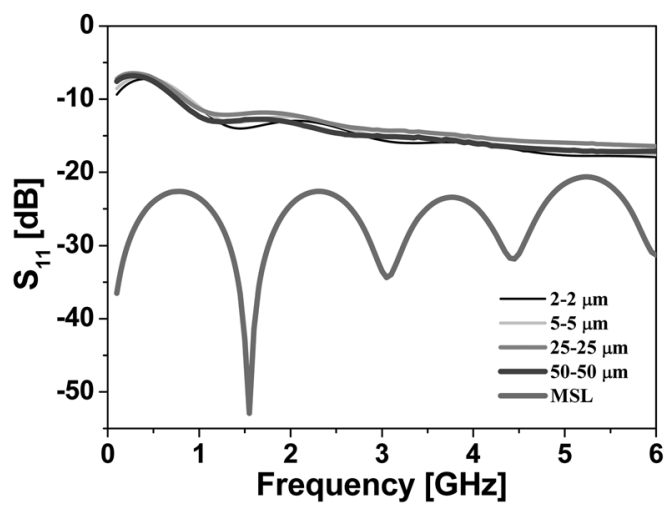

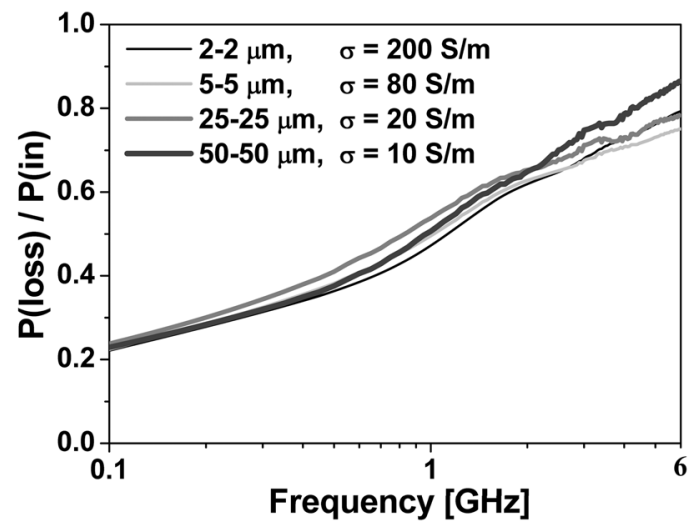

(a)

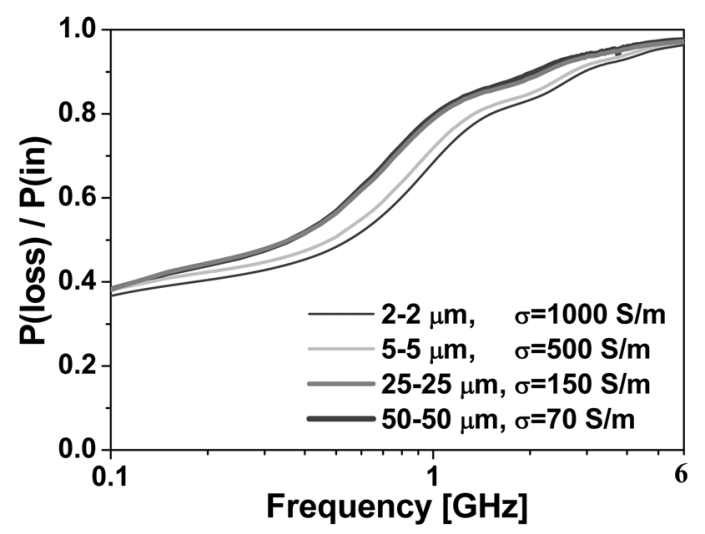

(b)

Fig. 6. Loss power properties of the double layered magnetic films with a reflection of (a) $5 \%$ and (b) $20 \%$ at various thicknesses.

reflections at 5 and $20 \%$ at the frequency of $0.3 \mathrm{GHz}$. The power loss of the magnetic films with a reflection of $20 \%$ increased strongly up to $68-79 \%$ and $96-98 \%$ at 1 and $6 \mathrm{GHz}$, respectively. On the other hand, with a reflection of $5 \%$, the power loss was $47-53 \%$ and 75-86 $\%$, respectively.

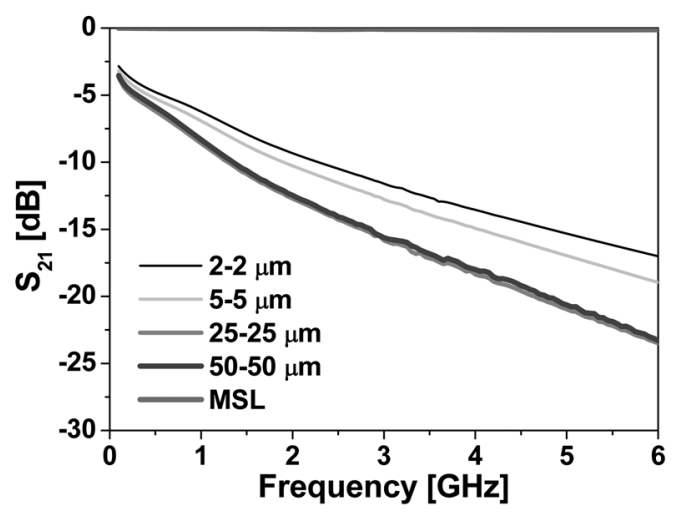

Fig. 7. Frequency response characteristics of the double layered magnetic films with a reflection of $20 \%$; (a) reflection loss and (b) transmission loss at various thicknesses. 
Fig. 7 shows the frequency dependence of the reflection and transmission loss of the magnetic films with different thicknesses and a reflection of $5 \%$ and $20 \%$, respectively. The reflection loss $\left(\mathrm{S}_{11}\right)$ was $-7.5 \sim-6.5 \mathrm{~dB}$ at 0.3 $\mathrm{GHz}$ at various thicknesses, while $\mathrm{S}_{11}$ was $<-10 \mathrm{~dB}$ in the frequency range from $1 \mathrm{GHz}$ to $6 \mathrm{GHz}$. It appears that the reflection loss was higher at frequencies $<0.3 \mathrm{GHz}$, but was still within the allowable value for practical use as an electromagnetic wave absorber [5]. Overall, a remarkable improvement in absorption performance of semiconducting/insulating layer magnetic films with broadband characteristics was achieved by controlling the conductivity up to an optimum level.

\section{Conclusion}

The loss power characteristics of the semiconducting/ insulating layer magnetic films with different levels of electrical conductivity on the microstrip line were examined by numerical analysis in quasi-microwave band. The remarkable improvement in absorbing performance was attributed to the increase in loss factor of the resistive loss. However, when the reflection was $>20 \%$, the reflection with increasing conductivity increased and the loss power deteriorated significantly. The numerical results of the magnetic field distribution confirmed that the radiated wave was emitted strongly with increasing conductivity of the semiconducting layer on the microstrip line due to the re-reflection of the radiated wave from the surface of the magnetic film. In conclusion, the high performance and broadband characteristics of the semiconducting/insulating layer magnetic films was achieved by controlling the conductivity and thickness to an optimum level.

\section{References}

[1] Y. Shimada, M. Yamaguchi, S. Ohnuma, T. Itoh, W. D. Li, S. Ikeda, K. H. Kim, and H. Nagura, "Granular Thin Films With High RF Permeability," IEEE Trans. Magn., 39, 3052-3056 (2003).

[2] K. Y. Kim, W. S. Kim, Y. D. Ju, and H. J. Jung, "Electromagnetic Wave Absorber of Laminated Ferrite and Dielectrics," Journal of the Korean Ceramic Society, 28(6), 483487 (1991).

[3] Y. W. Yun, S. W. Kim, G. Y. Kim, Y. B. Kim, Y. C. Yun, and K. S. Lee, "Electromagnetic shielding properties of soft magnetic metal and ferrite composites for application to suppress noise in a radio frequency range," Journal of Electroceramics, 17, 467-469 (2006).

[4] S. Yoshida, H. Ono, S. Ando, F. Tsuda, T. Itoh, Y. Shimada, M. Yamaguchi, K. I. Arai, S. Ohnuma, and T. Masumoto, "High frequency noise suppression in downsized circuits using magnetic granular films," IEEE Trans. Magn., 37, 2401-2403 (2001).

[5] H. Ono, T. Ito, S. Yoshida, Y. Takase, O. Hashimoto, and Y. Shimada, "Noble Magnetic Films for Effective Electromagnetic Noise Absorption in the Gigahertz Frequency Range,” IEEE Trans. Magn., 40, 2853-2857 (2004). 